\title{
Possible climate-related signals in high-resolution topography of lobate debris aprons in Tempe Terra, Mars
}

\author{
Peter M. Grindrod ${ }^{1,2}$ and Stephen A. Fawcett ${ }^{1,3}$ \\ Received 12 August 2011; revised 25 August 2011; accepted 30 August 2011; published 4 October 2011.
}

[1] Lobate debris aprons are common features in the midlatitudes of Mars that are assumed to be the result of the flow of ice-rich material. We produce high-resolution digital elevation models of two of these features in the Tempe Terra region of Mars using HiRISE stereo images. We identify two main topographic features of different wavelength using a power spectrum analysis approach. Short wavelength features, between approximately 10 and $20 \mathrm{~m}$ in length, correspond to a polygonal surface texture present throughout our study area. Long wavelength features, between approximately 700 and $1800 \mathrm{~m}$ in length, correspond to broad ridges that are up to $20 \mathrm{~m}$ in amplitude. We interpret both topographic signals to be the likely result of climate change affecting the debris contribution and/or the flow regime of the lobate debris aprons. The apparent surface age of about 300 Ma could be evidence of an astronomical forcing mechanism recorded in these lobate debris aprons at this time in Mars' history. Citation: Grindrod, P. M., and S. A. Fawcett (2011), Possible climate-related signals in high-resolution topography of lobate debris aprons in Tempe Terra, Mars, Geophys. Res. Lett., 38, L19201, doi:10.1029/2011GL049295.

\section{Introduction}

[2] Lobate debris aprons (LDAs) are amongst some of the most striking features on Mars thought to be related to nearsurface ice [e.g., Head et al., 2010]. LDAs are gently-sloping features that extend from or surround highland mesas and massifs, predominantly in the mid-latitudes, and often terminate at lobate margins [Squyres, 1979]. The apparent viscous flow morphologies and concentration in areas associated with near-surface ice suggest that LDAs are the result of the flow of a combination of ice and rock [e.g., Squyres, 1979; Colaprete and Jakosky, 1998; Mangold and Allemand, 2001; Head et al., 2005]. The sensitivity of nearsurface ice to fluctuations in climate conditions means that LDAs have the ability to record past climate conditions on Mars [e.g., Kreslavsky et al., 2008]. Here we attempt to exploit this sensitivity by studying the high-resolution topography of two ridged LDAs near the Martian crustal dichotomy boundary in Tempe Terra.

\footnotetext{
${ }^{1}$ Department of Earth Sciences, University College London, London, UK. UK.

${ }^{2}$ Centre for Planetary Sciences, University College London, London,

${ }^{3}$ Now at National Oceanography Centre, Southampton, UK.
}

Copyright 2011 by the American Geophysical Union. 0094-8276/11/2011GL049295

\section{Topography of the Lobate Debris Aprons}

\subsection{Topography and Slope}

[3] The two LDAs in this study, hereafter North and South LDAs, have overall topographic shapes typical of other LDAs in Tempe Terra [e.g., Chuang and Crown, 2005] and elsewhere on Mars [e.g., Mangold and Allemand, 2001]. The LDAs surround and lie at the base of isolated and relatively steep central massifs, whose summits are between 2 and $2.5 \mathrm{~km}$ above the surrounding terrain (Figure 1). The LDAs extend radially up to about 4 or $5 \mathrm{~km}$ from the central massifs, with distance varying around the deposits. The North and South LDAs have maximum elevations of approximately -3110 and $-3010 \mathrm{~m}$ respectively, and minimum elevations of about -3490 and $-3370 \mathrm{~m}$ respectively, giving an overall elevation difference of about $360-380 \mathrm{~m}$. The overall mean slopes are about $4-4.5^{\circ}$, increasing slightly towards the lower apron slopes giving an overall convex-up profile, common to other LDAs in Tempe Terra [Chuang and Crown, 2005].

[4] Our HiRISE stereo DEMs reveal deviations from the standard convex-up profile at both LDAs in the study area. The detrended elevations (see auxiliary material) show ridges in the surface of the LDAs that have amplitudes of up to about $20 \mathrm{~m}$, which decrease in height with increasing distance from the massif (Figures $2 a$ and $2 b$ ). The ridges in the North LDA have a striking periodicity, with a wavelength of between 600 to $1000 \mathrm{~m}$ from visual inspection. The periodicity is less well defined in the South LDA, but there is still an apparent repeating wavelength of about 1000 to $1500 \mathrm{~m}$ from visual inspection. These ridges are also evident in detrended slope profiles (Figures $2 \mathrm{c}$ and $2 \mathrm{~d}$ ). The slope profiles mimic the periodicity of the elevation profiles, but also show significant variation over much shorter distances, with changes in slope often greater than $5^{\circ}$ over a distance of 10-20 m. The number of ridges identified at each LDA varies, but there are at least 5 and 3 ridge and trough sequences at the North and South LDAs respectively.

\subsection{Power Spectrum Analysis}

[5] To determine the exact lengthscales of the ridges in the LDA profiles, we analyzed the power spectrum of topographic slope profiles (see auxiliary material)-a method that has been previously used to identify quasi-periodic bedding in sedimentary sequences on Mars [Lewis et al., 2008]. We identified two main frequencies of spectral peaks at both the North and South LDAs (Figures 2e and 2f) that exceeded the critical confidence level. Long wavelength periodic signals occur at $678-1055 \mathrm{~m}$ and $1151-1809 \mathrm{~m}$ at the North and South LDAs respectively. Short wavelength periodic signals are also identified at $10-16 \mathrm{~m}$ and 9-20 m at the North and South LDAs respectively. Short wave- 

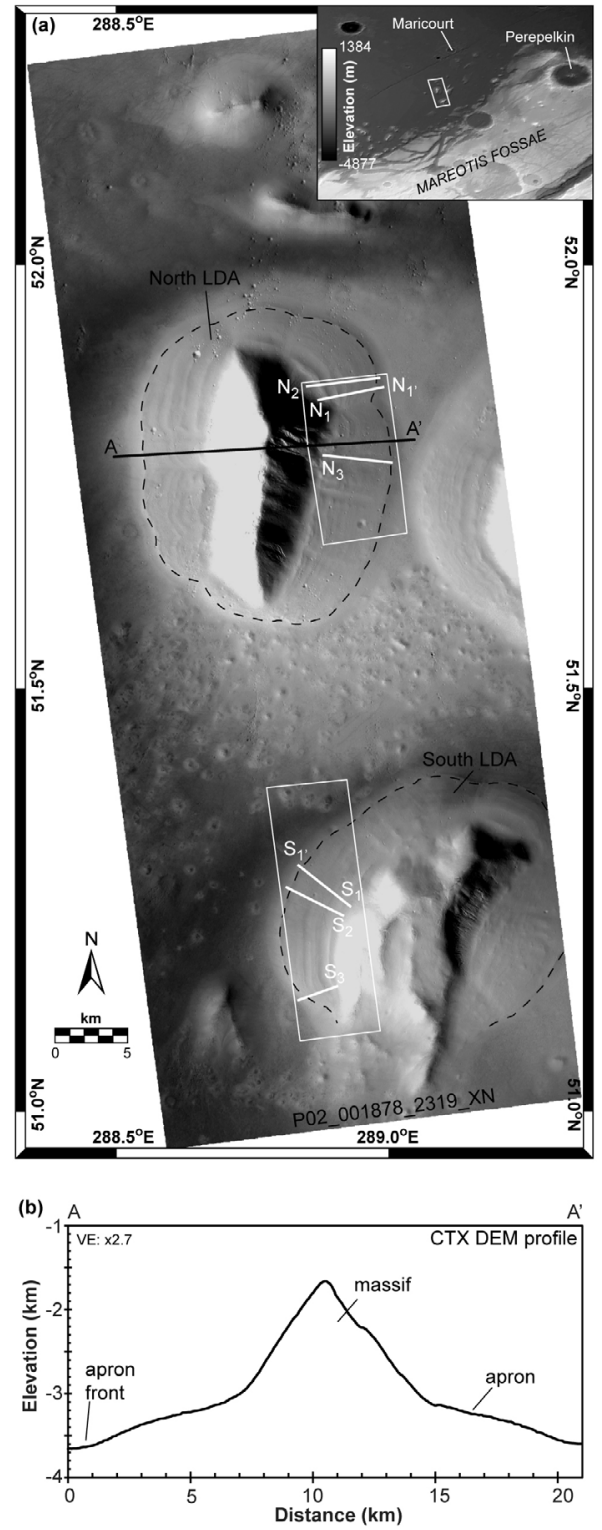

Figure 1. Location of the study area. (a) Inset shows a colorized MOLA hillshade of the general study region, to the north-west of Tempe Terra/Mareotis Fossae, and the location of the main context image (white box). Main image is a $6 \mathrm{~m} / \mathrm{px}$ CTX image. Dashed lines show the approximate location of the edge of the North and South LDAs, and the white boxes show the location of the HiRISE stereo DEMs made at $1 \mathrm{~m} / \mathrm{px}$. Solid black line shows a topographic profile through the North LDA using the CTX DEM. Solid white lines show topographic profiles using the HiRISE DEMs. (b) Topographic profile through the North LDA using the CTX DEM, showing the general shape including central massif, the apron region and apron front.

length signals of 1-4 m also exceed the critical confidence level but are attributed to noise at the scale of the slope input signal $(3 \times 3$ pixels of $1 \mathrm{~m}$ square are averaged to determine the slope value). To ensure that the periodic signals occurred throughout the LDAs and not just our profile locations, we repeated this technique with profiles in a further two loca- tions taken at both the North and South LDAs (Figure 1). The results of these power spectrum analyses were similar, showing dual wavelengths of periodicity (Figure 3a): short wavelengths of about 10-20 m, and long wavelengths of about $700-1800 \mathrm{~m}$.

\section{Discussion and Implications}

[6] Here we discuss the possible formation mechanisms of both the short and long wavelength topographic signals, in order to determine their combined implications for recent Martian ice processes and climate history. The surfaces of the LDAs have a polygonal texture (Figure 3b), which is common at LDAs [e.g., Mangold, 2005], and the short wavelength $(\sim 10-20 \mathrm{~m})$ periodic features in our power spectrum analyses correspond to the dimensions of these polygons. Inspection of both the spectral plots and fullresolution HiRISE images suggests that there is no bimodal distribution of polygon dimensions, as observed in 'basketball terrain' [e.g., Marchant et al., 2002], but instead a relatively homogenous size range that corresponds to the small polygon type of Mangold [2005]. This unimodal size distribution suggests that the patterned surface at our study LDAs records a common formation mechanism and/or period of polygonal terrain formation [Mangold, 2005]. Polygonal features of this scale are common on both Earth and Mars in areas that are associated with ice-rich material in the upper few meters of the terrain [e.g., Marchant et al., 2002] and, regardless of the exact formation method (e.g., thermal contraction, sublimation, freeze-thaw), are often interpreted as evidence of cyclic processes [e.g., Kessler and Werner, 2003]. Both thermal contraction and freeze-thaw methods of polygon formation require repeated cycles of activity, and on Mars have been invoked over both seasonal timescales and periods of changing obliquity and eccentricity depending on size and formation method [e.g., Mangold, 2005; Marchant and Head, 2007]. The efficacy of sublimation of pre-fractured terrain [Mangold, 2003] and subsequent partial burial by mantling material [Mustard et al., 2001] to form quasi-polygonal features would also vary with changing climatic conditions. It is likely that these small polygonal features represent a different climate period in the Late Amazonian, probably in the last $10 \mathrm{Ma}$ or later. This idea is supported by estimates of the abundance and depth of ice deposits in the broader Tempe Terra region, which are expected to enhance polygon processes during times of high obliquity [e.g., Chamberlain and Boynton, 2007].

[7] The ridges in the North LDA were identified and discussed using earlier data sets by Chuang and Crown [2005]. Our power spectrum analyses reveal ridge wavelengths of approximately $680-1050 \mathrm{~m}$ at the North LDA, which matches well with the 400-800 m wavelengths previously identified at this LDA using lower resolution imagery alone [Chuang and Crown, 2005]. This similarity not only adds confidence to the power spectrum method, but also confirms that the ridges do have a topographic signature that can be studied in HiRISE DEMs. Chuang and Crown [2005] identified 11 massif-related LDAs in the Tempe Terra/Mareotis region that show evidence in imagery alone of multiple broad ridges, suggesting that a common process or processes could be responsible for ridge formation in the LDAs in at least this region. 

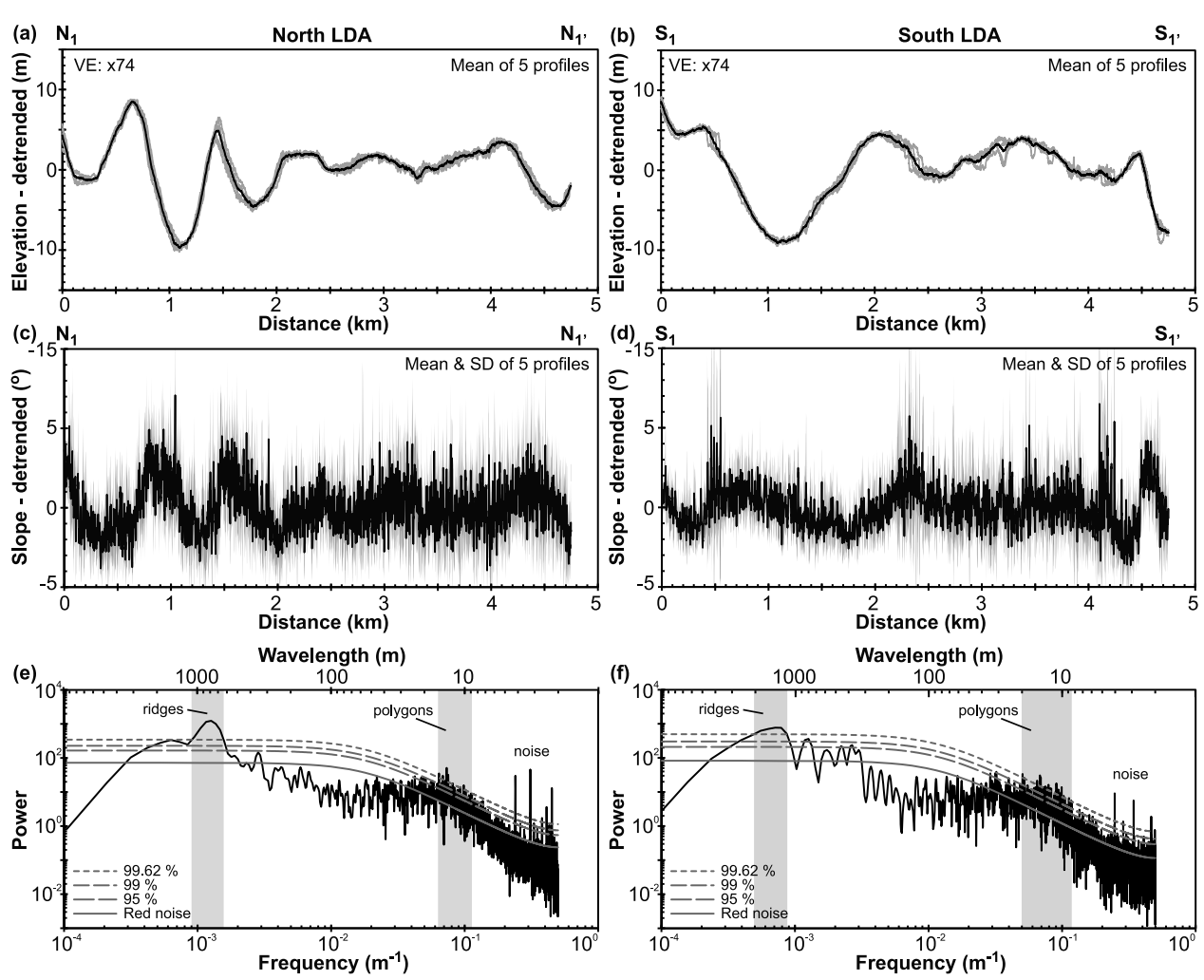

Figure 2. Results of the topographic analysis. ( $\mathrm{a}$ and $\mathrm{b}$ ) The mean (solid black line) of 5 detrended topographic profiles (gray lines) through the North and South LDAs using the HiRISE DEMs. (c and d) The mean (solid black line) and standard deviation (gray lines) of 5 detrended slope profiles through the North and South LDAs, corresponding with those profiles in Figures $2 a$ and $2 b$. (e and f) The power spectra of the slope profiles of the North and South LDAs respectively. Gray lines represent estimated red noise (solid) and confidence levels (dashed) of 95, 99 and 99.62\%. Shaded regions represent those wavelengths where the power spectra exceed the critical (99.62\%) confidence levels.

[8] Two possible methods of ridge formation have previously been proposed: (1) changes in debris production, and (2) changes in flow properties [Chuang and Crown, 2005]. We expand upon these explanations and add the expression of the underlying bedrock topography as a third possible mechanism. As the LDAs lie downslope of a central rock massif, it is likely that debris material has been delivered to the LDA from the massif. If the rate of debris production has fluctuated due to a change in the rate of massif erosion then the topography of the LDA could reflect this change. The ridges could be evidence of increased debris production from the massifs during a period of constant flow of the LDAs [Chuang and Crown, 2005]. This explanation is supported at the North LDA by the presence of three gully systems with distinctive fan deposits each at least $0.5 \mathrm{~km}^{2}$ that merge into the LDA. Polygons are present on some, but not all, of the gully material, indicating overlapping formation times of at least some of these feature types. There is mounting evidence that gully formation might be related to the recent climate history of Mars, regardless of their exact formation mechanism, varying as a result of obliquity-related climate change [e.g., Levy et al., 2011]. Differential sublimation of overlapping LDA flows, the result of periodic massif erosion and debris contribution, could also lead to the formation of ridge and trough topography. It is therefore possible that the multiple ridges represent repeated episodes of fluctuating massif erosion and debris deposition within the LDAs.
[9] It is also possible that the ridge and trough topography is the result of changes in the flow properties of the LDAs. Depending on the amount of ice present, the bulk viscosity of the LDAs is likely to vary as a function of a number of factors, including the dust/rock content, temperature, grain size, and applied stress. Any change in the surrounding climate, if operating over sufficient timescales, has the potential to cause significant changes in the rheology-controlling parameters and thus in the flow behavior of an LDA. The viscosity of an LDA could be reduced by a temperature increase and/or a reduction in the dust content, which in turn could lead to accelerated flow in the LDA and possible thinning. This scenario is an example of periodic non-steady flow in the LDA, represented at the surface as a kinematic wave [e.g., Paterson, 1994]. Surface 'waves' have been observed at the Byrd Glacier in Antarctica, which are most likely the result of changes in glacier-bed coupling associated with the transition from ice sheet to shelf [e.g., Hughes, 2009]. LDAs on Mars lack the boundary conditions associated with ice streams and grounding lines, and alternative methods must be invoked for the observed surface ridges. An increase in ice thickness and the appearance of topographic waves has also been observed in terrestrial glaciers during periods of surging and compression folding [e.g., Dolgushin and Osipova, 1975]. Glacier surging is likely due to a combination of internal mechanisms and factors, such as bed deformation or draining, rather than the surrounding climate [e.g., Murray et al., 2003]. However, surging often involves 

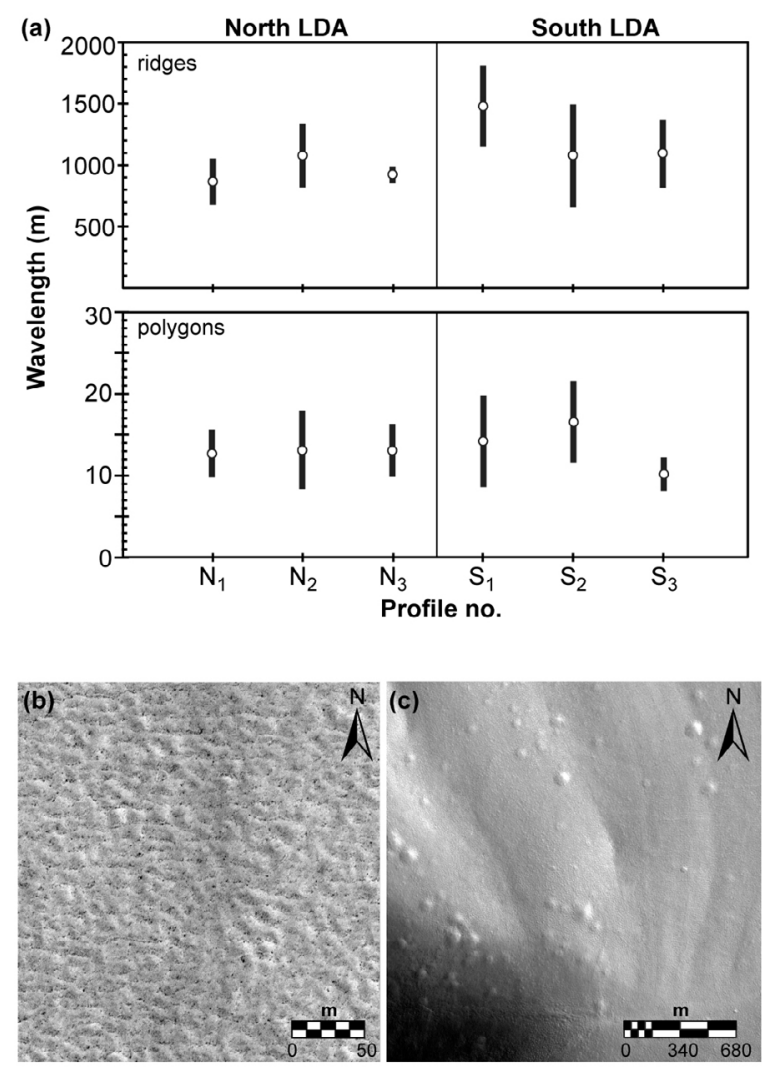

Figure 3. (a) The two scales of topographic variation in the LDAs. The mean (circle) and range (black bars) of wavelengths at which the power spectra exceed the critical confidence level are shown for each profile. Profiles $\mathrm{N}_{1}$ and $\mathrm{S}_{1}$ are those shown in Figure 2. (b) Polygonal texture of the LDA surface that corresponds with the short wavelength periodic features. Image is a subset of PSP_008563_2320, approximately half way along profile $\mathrm{N}_{1}-\mathrm{N}_{1}{ }^{\prime}$ in Figures 1 . (c) Ridge and trough topography evident in the North LDA, in HiRISE image PSP_008563_2320. Apparent flow direction, and illumination is approximately from left to right in this image.

the glacier velocity increasing by 10-1000 times [Murray et al., 2003], which leads to fast strain rates and the brittle failure of ice manifested through high densities of crevasses. We see little evidence for crevasses at our LDAs, which is suggestive of little to no brittle failure associated with ridge formation and thus low strain rates. However, some linear features $\sim 5-10 \mathrm{~m}$ wide occurring in both the North and South LDA, which are oriented both perpendicular and parallel to the apparent flow direction and have some similarities to the cracks identified by Hubbard et al. [2011], could be evidence of some brittle processes and subsequent infilling.

[10] Thirdly, the steady-state flow of glaciers over undulating bedrock can result in variations in the stress and velocity fields creating surface perturbations [e.g., Gudmundsson, 2003]. In this case, the longest and shortest bedrock wavelengths are damped and not transferred to the surface of a glacier, instead intermediate wavelengths of about 3-5 times the ice thickness are observed [Hutter et al., 1981]. This would require LDA thicknesses of up to about $300 \mathrm{~m}$ based on the ridge wavelengths, which seems reasonable given likely LDA thicknesses observed by SHARAD [Plaut et al., 2009]. Maximum slopes at the surface would then correspond to bedrock highs. Although this mechanism cannot be ruled out as the source of the ridges, the observation of similar ridges at a number of LDAs throughout the Tempe Terra region [Chuang and Crown, 2005] requires similar bedrock topography at all of the corresponding central massifs.

[11] Overall, the constant unifying theme running through our analysis is that of climate change. Although it is not possible to definitively identify the cause of the ridges at our study LDAs, the most plausible mechanisms can all be activated and/or enhanced through repeated changes in the climate. Recent detailed geomorphological studies of LDAs have highlighted the likely cyclical nature of processes controlling mass loss at LDAs [Baker et al., 2010; Hubbard et al., 2011]. The approximate 100:1 size ratio of polygons and ridges could correspond to the obliquity timescales of Mars [Laskar et al., 2004], and represent similar periods of cyclic behavior to those observed in some sedimentary beds on Mars [Lewis et al., 2008]. Given a crater count-derived age of $\sim 300 \mathrm{Ma}$ for our LDAs (see auxiliary material), then these features could provide evidence of periodic climate change further into the past than identified in previous studies. If climate is the key factor in causing the topographic features at our study LDAs then it could offer constraints on the response time of these ice-rich features to climate variations in this region, and possibly elsewhere on Mars.

[12] Acknowledgments. PMG is funded by an STFC Aurora Research Fellowship (grant ST/F011830/1). We thank James Scotchman for advice on time series analysis methods, and Reid Parsons and an anonymous reviewer for comments that greatly improved the clarity and robustness of the study. We also thank the CTX and HiRISE teams for making the data available, and providing tools to aid in their processing. The stereo DEM processing was carried out at the UK NASA RPIF at University College London.

[13] The Editor thanks Reid Parsons and an anonymous reviewer for their assistance in evaluating this paper.

\section{References}

Baker, D. M. H., J. W. Head, and D. R. Marchant (2010), Flow patterns of lobate debris aprons and lineated valley fill north of Ismeniae Fossae, Mars: Evidence for extensive mid-latitude glaciations in the Late Amazonian, Icarus, 207, 186-209, doi:10.1016/j.icarus.2009.11.017.

Chamberlain, M. A., and W. V. Boynton (2007), Response of Martian ground ice to orbit-induced climate change, J. Geophys. Res., 112, E06009, doi:10.1029/2006JE002801

Chuang, F. C., and D. C. Crown (2005), Surface characteristics and degradational history of debris aprons in the Tempe Terra/Mareotis Fossae region of Mars, Icarus, 179, 24-42, doi:10.1016/j.icarus.2005.05.014.

Colaprete, A., and B. M. Jakosky (1998), Ice flow and rock glaciers on Mars, J. Geophys. Res., 103, 5897-5909, doi:10.1029/97JE03371.

Dolgushin, L. D., and G. B. Osipova (1975), Glacier surges and the problem of their forecasting, in Snow and Ice, IAHS Publ., 104, 292-304.

Gudmundsson, G. H. (2003), Transmission of basal variability to a glacier surface, J. Geophys. Res., 108(B5), 2253, doi:10.1029/2002JB002107.

Head, J., et al. (2005), Tropical to mid-latitude snow and ice accumulation, flow, and glaciations on Mars, Nature, 434, 346-351, doi:10.1038/ nature 03359

Head, J. W., D. R. Marchant, J. L. Dickson, A. M. Kress, and D. M. Baker (2010), Northern mid-latitude glaciation in the Late Amazonian period of Mars: Criteria for the recognition of debris-covered glacier and valley glacier landsystem deposits, Earth Planet. Sci. Lett., 294, 306-320, doi:10.1016/j.eps1.2009.06.041

Hubbard, B., R. E. Milliken, J. S. Kargel, A. Limaye, and C. Souness (2011), Geomorphological characterization and interpretation of a midlatitude glacier-like form: Hellas Planitia, Mars, Icarus, 211, 330-346, doi:10.1016/j.icarus.2010.10.021. 
Hughes, T. (2009), Variations of ice bed coupling beneath and beyond ice streams: The force balance, J. Geophys. Res., 114, B01410, doi:10.1029/ 2008JB005714.

Hutter, K., F. Legerer, and U. Spring (1981), First-order stresses and deformations in glaciers and ice sheets, J. Glaciol., 27, 227-270.

Kessler, M. A., and B. T. Werner (2003), Self-organization of sorted patterned ground, Science, 299, 380-383, doi:10.1126/science.1077309.

Kreslavsky, M. A., J. W. Head, and D. R. Marchant (2008), Periods of active permafrost layer formation during the geological history of Mars: Implications for circum-polar and mid-latitude surface processes, Planet. Space Sci., 56, 289-302, doi:10.1016/j.pss.2006.02.010.

Laskar, J., A. C. M. Correia, M. Gastineau, F. Joutel, B. Levrard, and P. Robutel (2004), Long term evolution and chaotic diffusion of the insolation quantities of Mars, Icarus, 170, 343-364, doi:10.1016/j.icarus. 2004.04.005.

Levy, J. S., J. W. Head, and D. R. Marchant (2011), Gullies, polygons and mantles in Martian permafrost environments: Cold desert landforms and sedimentary processes during recent Martian geological history, in IceMarginal and Periglacial Processes and Sediments, edited I. P. Martini, H. M. French, and A. Pérez Alberti, Geol. Soc. Spec. Publ., 354, 167-182, doi:10.1144/SP354.10.

Lewis, K. W., O. Aharonson, J. P. Grotzinger, R. L. Kirk, A. S. McEwen, and T.-A. Suer (2008), Quasi-periodic bedding in the sedimentary rock record of Mars, Science, 322, 1532-1535, doi:10.1126/science.1161870.

Mangold, N. (2003), Geomorphic analysis of lobate debris aprons on Mars at Mars Orbiter Camera scale: Evidence for ice sublimation initiated by fractures, J. Geophys. Res., 108(E4), 8021, doi:10.1029/2002JE001885.

Mangold, N. (2005), High latitude patterned grounds on Mars: Classification, distribution and climatic control, Icarus, 174, 336-359, doi:10.1016/j.icarus. 2004.07.030.

Mangold, N., and P. Allemand (2001), Topographic analysis of features related to ice on Mars, Geophys. Res. Lett., 28, 407-411, doi:10.1029/ 2000GL008491.
Marchant, D. R., and J. W. Head (2007), Antarctic dry valleys: Microclimate zonation, variable geomorphic processes, and implications for assessing climate change on Mars, Icarus, 192, 187-222, doi:10.1016/ j.icarus.2007.06.018.

Marchant, D. R., A. Lewis, W. C. Phillips, E. J. Moore, R. Souchez, and G. P. Landis (2002), Formation of patterned-ground and sublimation till over Miocene glacier ice in Beacon Valley, Antarctica, Geol. Soc. Am. Bull. 114, 718-730, doi:10.1130/0016-7606(2002)114<0718:FOPGAS > 2.0. $\mathrm{CO} ; 2$.

Murray, T., T. Strozzi, A. Luckman, H. Jiskoot, and P. Christakos (2003), Is there a single surge mechanism? Contrasts in dynamics between glacier surges in Svalbard and other regions, J. Geophys. Res., 108(B5), 2237, doi:10.1029/2002JB001906.

Mustard, J. F., C. D. Cooper, and M. K. Rifkin (2001), Evidence for recent climate change on Mars from the identification of youthful near-surface ground ice, Nature, 412, 411-414, doi:10.1038/35086515.

Paterson, W. S. B. (1994), The Physics of Glaciers, Butterworth-Heinemann, Oxford, U. K.

Plaut, J. J., A. Safaeinili, J. W. Holt, R. J. Phillips, J. W. Head, R. Seu, N. E. Putzig, and A. Frigeri (2009), Radar evidence for ice in lobate debris aprons in the mid-northern latitudes of Mars, Geophys. Res. Lett., 36, L02203, doi:10.1029/2008GL036379.

Squyres, S. W. (1979), The distribution of lobate debris aprons and similar flows on Mars, J. Geophys. Res., 84, 8087-8096, doi:10.1029/ JB084iB14p08087.

S. A. Fawcett, National Oceanography Centre, European Way, Southampton SO14 3ZH, UK.

P. M. Grindrod, Department of Earth Sciences, University College London, Gower Street, London WC1E 6BT, UK. (p.grindrod@ucl.ac.uk) 